\title{
PRODUÇÃO FOTOSSINTÉTICA DA MACROALGA ULVA LACTUCA LINNAEUS (CHLOROPHYCEAE) CULTIVADA EM EFLUENTES DE UMA FAZENDA DE CAMARÕES MARINHOS.
}

\author{
ALENCAR, J. R. ${ }^{\text {; }}$ HORTA JUNIOR, P. A. ${ }^{2} \&$ CELINO, J. J. ${ }^{3 *}$ \\ 1 - Rua Vitória Régia, 124. Córrego Grande - CEP 88037-130 - Florianópolis - Santa Catarina - Brasil, \\ e_mail: jefferson.alencar@gmail.com \\ 2 - Laboratório de Ficologia. Universidade Federal de Santa Catarina, Centro de Ciências Biológi- \\ cas, Departamento de Botânica. Trindade - CEP 88010-970 - Florianópolis, Santa Catarina - Brasil, \\ e_mail: pahorta@ufsc.br \\ 3 - Núcleo de Estudos Ambientais, Instituto de Geociências, Universidade Federal da Bahia (UFBA), \\ Rua Barão de Geremoabo, s/n, Sala 304 A1, Federação - CEP 40170-290 - Salvador - Bahia - Brasil. \\ *Corresponding author: joil@ufba.br
}

\begin{abstract}
Alencar, J. R.; Horta Junior, P. A. \& Celino, J. J. 2010. Photosynthetic production of the macroalgae Ulva lactuca Linnaeus (Chlorophyceae) cultivated in wastewater from a shrimp marine farm. Braz. J. Aquat. Sci. Technol. 14(2):55-62. ISSN 1808-7035. In order to assess the demand for oxygen by photosynthetic production of the macroalgae Ulva lactuca grown in effluent from a shrimp farm and point out the potential growth of this species integrated closed recirculation systems, specimens were collected in Lagoa da Conceição - Florianópolis (SC), Brazil and transported to the laboratory to check the stems. The experiment used four treatments with seaweed and algae with no one, with the following densities of algal material: $1=1 \mathrm{~g} \mathrm{~L}^{-1}, 2=2 \mathrm{~g} \mathrm{~L}^{-1}, 3=3 \mathrm{~g} \mathrm{~L}^{-1}$ and $4=4 \mathrm{~g} \mathrm{~L}^{-1}$. Supersaturation of oxygen generated by photosynthetic activity of macroalgae far surpassed the rate of breathing. Lower densities between 1 and $2 \mathrm{gL}^{-1}$, and provide high levels of oxygen during the day, consume proportionally little above the gas at night, favoring the maintenance of the stems and represent a great potential for integrated cultivation to shrimp farming in marine recirculation system, since that is situated in a basin of stabilization.
\end{abstract}

Keywords: Litopenaeus vannamei; photoinhibition; closed system of recirculation

\section{INTRODUÇÃO}

A fotossíntese em macroalgas clorófitas ocorre de forma semelhante ao mecanismo das plantas $\mathrm{C}_{3}$, onde a luz absorvida pelo conjunto de moléculas das clorofilas a e $b$ possibilita a oxidação da molécula de água em oxigênio, convertendo energia luminosa em energia química e gerando a transferência de elétrons do Fotossistema II para o Fotossistema I, através da cadeia transportadora de elétrons (Salisbury \& Ross, 1992). Na fotossíntese, tais macroalgas processam o $\mathrm{CO}_{2}$ diretamente, através da enzima ribulose-1,5 bisfosfato carboxilase (Rubisco) nas células dos talos, produzindo um açúcar de três carbonos. A enzima Rubisco, além da atividade carboxilase, também apresenta atividade oxigenase, realizando o processo de fotorrespiração. Os Fotossistemas I e II, apesar de serem estruturalmente diferentes, cooperam entre si para a produção fotossintética, onde o primeiro requer comprimentos de onda inferiores a 700nm e o segundo, inferiores a 680nm (Kirk, 1994). Segundo Werlinger \& Alveal (1996), quando a faixa de intensidade luminosa ultrapassa o ponto de saturação e de fotossíntese máxima, ocorre o processo de fotoinibição, produzindo a inativação do aparelho fotossintético.
De acordo com Kubitza (2003), é a partir da atividade fotossintética que as macroalgas geram energia e sintetizam outros compostos dissolvidos na água, os quais são importantes para o seu crescimento e reprodução. Quando a fotossíntese supera a respiração por períodos prolongados, pode estar ocorrendo um excesso de material orgânico no sistema. Coutinho \& Zingmark (1993) relataram que a macroalga Ulva curvata pode apresentar uma produção fotossintética superior à sua demanda respiratória. King \& Schramm (1976), Lapointe \& Tenore (1981) e Lapointe \& Duke (1984) verificaram que o crescimento e a fotossíntese das macroalgas são regulados pelo nitrogênio inorgânico e pela luz.

Entretanto, o crescimento, o desenvolvimento e a permanência de espécies e comunidades algais dependem da interação com variáveis biológicas e ambientais. No ambiente natural, as macroalgas marinhas bênticas se distribuem desde o supralitoral até a profundidade onde a iluminação é suficiente para o processo fotossintético (Werlinger \& Alveal, 1996). Além da intensidade luminosa, outras condicionantes ambientais, como a turbulência, a variação de temperatura e de salinidade, podem interferir na produção fotossintética das macroalgas. Segundo Vergara et al. 
(1997), a ressuspensão de sedimentos e a sobreposição da biomassa algal podem levar a uma considerável atenuação da luz para o processo fotossintético. Em Ulva rotundata, elevadas temperaturas induzem, a curto prazo, ao aumento da atividade fotossintética e respiratória da alga (Henley, 1992). Lartigue et al. (2003), observaram que a macroalga $U$. lactuca alcança melhores taxas fotossintéticas em salinidades mais baixas, sendo a faixa ótima de $25 \mathrm{ppt}$.

Para Floreto et al. (1993), a relação da demanda de nutrientes, intensidade luminosa, temperatura e baixa salinidade são fatores que contribuem no crescimento de algas do gênero Ulva, tornando-as parceiras potenciais em cultivos integrados à carcinicultura marinha. Abundante no litoral brasileiro, U. lactuca pode otimizar, através de sistema fechado de recirculação, a disponibilidade de oxigênio nos viveiros de camarões marinhos. Em Kubitza (2003), a fotossíntese supre cerca de 50 a 95\% de todo o oxigênio disponível em viveiros com baixa renovação de água. Uma técnica usada para quantificar as propriedades ecofisiológicas da fotossíntese algal é a determinação das mudanças de oxigênio através de um monitoramento (Cabello-Pasini et al., 2000; Häder et al., 2000 e Levy et al., 2004).

Para Villarreal et al. (1994), o limite mínimo de $\mathrm{O}_{2}$ para a sobrevivência do camarão marinho Litopenaeus vannamei (Boone, 1931) é de $1,3 \mathrm{mg}$ de $\mathrm{O}_{2}$ $\mathrm{L}^{-1}$. De acordo com estes autores, o oxigênio é considerado um dos parâmetros fundamentais em cultivos, fazendo parte dos processos metabólicos do animal, e está condicionado aos efeitos da temperatura e da salinidade. Por exemplo, em temperatura de $26^{\circ} \mathrm{C}$ e salinidade de 32ppt, a solubilidade do oxigênio é de $6,76 \mathrm{mg}$ de $\mathrm{O}_{2} \mathrm{~L}^{-1}$ (Kubitza, 2003). Em fazendas com cultivo intensivo desta espécie e baixo potencial eólico, os gastos com energia elétrica para manter aeradores em funcionamento, objetivando sustentar faixas confortáveis de oxigênio dissolvido (acima de $4 \mathrm{mg}$ de $\mathrm{O}_{2} \mathrm{~L}-$ $\left.{ }^{1}\right)$ para os camarões, acabam elevando os custos de produção.

Este estudo teve como objetivo avaliar a demanda respiratória e a produção de oxigênio disponibilizado pela produção fotossintética da macroalga U. lactuca, cultivada em efluentes do cultivo de camarões. Considerando o oxigênio dissolvido um dos parâmetros fundamentais para o cultivo de camarões marinhos da espécie L. vannamei, este trabalho busca, portanto, averiguar o potencial de cultivo desta macroalga, através do consumo de nutrientes para fornecer oxigênio, integrada às fazendas de camarões marinhos, em diferentes horários do dia e seus respectivos picos, assim como verificar se os efluentes são bons ou não para o crescimento da macroalga.

\section{MATERIAIS E MÉTODOS}

\section{Coleta e preparo do material biológico}

O presente experimento foi realizado no Laboratório de Ficologia Aplicada (LAFICA), situado no Departamento de Aqüicultura, Centro de Ciências Agrárias, Universidade Federal de Santa Catarina, Florianópolis, sul do Brasil. Ulva lactuca foi coletada nos tanques de sedimentação da Estação de Piscicultura Marinha da Universidade Federal de Santa Catarina, junto à Lagoa da Conceição, localizado aproximadamente na latitude $27^{\circ} 30^{\prime} \mathrm{S}$ e longitude $48^{\circ} 30^{\prime} \mathrm{W}$.

O material coletado foi transportado em bandejas, sem água, até o LAFICA. Em seguida, foi previamente lavado em água doce corrente para eliminação de organismos indesejáveis. Os talos foram selecionados, destacando-se vários discos com diâmetro de $5 \mathrm{~cm}$. Para a determinação do peso úmido, os discos foram comprimidos em papel absorvente e pesados em balança eletrônica, marca Marte, mod. AL500.

\section{Delineamento experimental}

Para o delineamento experimental, foram utilizados 30 frascos Erlenmeyer de $500 \mathrm{~mL}$, previamente lavados em uma solução de água destilada com $10 \%$ de ácido clorídrico e enxaguados abundantemente com água destilada. Cada frasco recebeu $500 \mathrm{~mL}$ de água do canal de despesca da Fazenda Batavia, situada no município de Biguaçu, distante $30 \mathrm{~km}$ ao norte de Florianópolis, onde se cultivam camarões marinhos da espécie Litopenaeus vannamei. A água coletada apresentou uma salinidade de 32 e quantidades médias de amônia e ortofosfato em torno de $40 \mu \mathrm{g} \cdot \mathrm{L}^{-1}$ e $5 \mu \mathrm{g} \cdot \mathrm{L}^{-1}$, respectivamente.

O experimento contou dos quatro tratamentos com alga em diferentes densidades e um sem alga, bem como de seus respectivos controles escuros. Todos os tratamentos foram compostos de três repetições (Fig.1A). Os recipientes controle foram envolvidos com papel laminado para bloquear a ação da luz. Os tratamentos foram separados em quatro diferentes densidades ( $1=1 \mathrm{~g} \mathrm{~L}^{-1}, 2=2 \mathrm{~g} \mathrm{~L}^{-1}, 3=3 \mathrm{~g} \mathrm{~L}^{-1}$ e $4=4 \mathrm{~g} \mathrm{~L}^{-1}$ ) recebendo as biomassas aproximadas de $0,5 \mathrm{~g}, 1 \mathrm{~g}, 1,5 \mathrm{~g}$ e $2 \mathrm{~g}$ de alga, respectivamente, ajustando-se à capacidade dos recipientes. Em seguida, estes frascos foram cobertos com uma película transparente de PVC e protegidos com papel alumínio até o início do experimento.

Em um tanque plástico, de seção circular, com capacidade de 1000L e pleno de água, os tratamentos foram distribuídos ao acaso sobre uma mesa submersa (Fig. 1B). Apenas $5 \mathrm{~cm}$ da parte superior dos frascos de Erlenmeyer ficaram fora d'água. O experimento foi mantido sob renovação periódica de água, armazenada em reservatório subterrâneo, com o objetivo de conservar a 


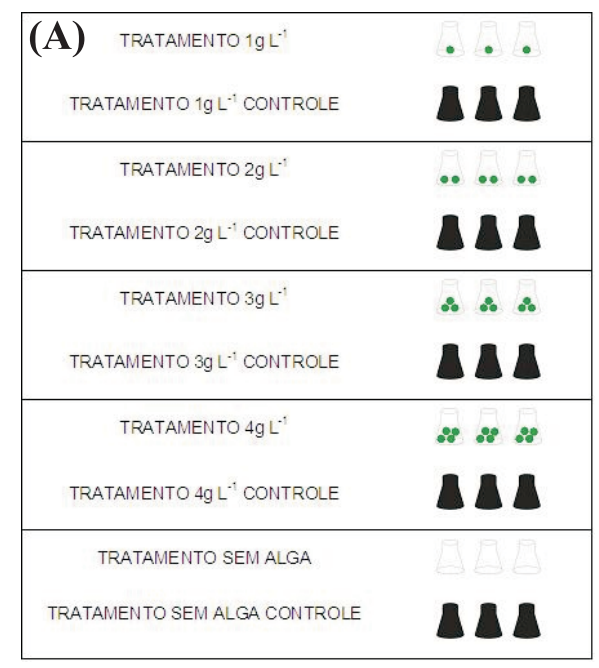

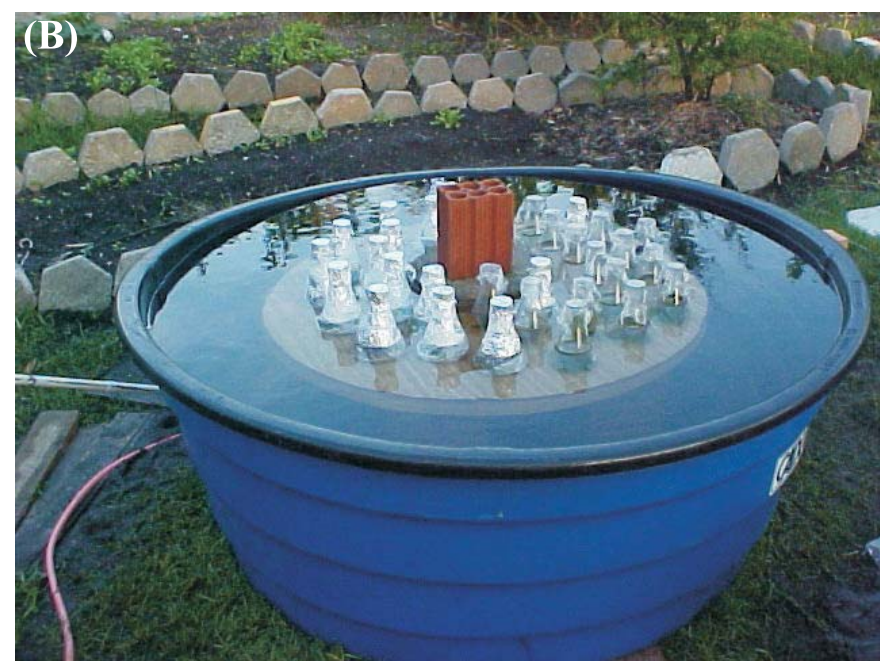

Figura1 - (A) Esquema do delineamento experimental. Os quatro primeiros tratamentos com suas densidades algais e seus respectivos controles. O último tratamento, sem alga, e o seu controle. (B) Delineamento experimental mostrando a distribuição dos frascos de Erlenmayer cobertos com papel alumínio, antes do início do experimento.

temperatura estável $\left(26 \pm 1^{\circ} \mathrm{C}\right)$. Do centro do delineamento experimental, a água foi distribuída regularmente, de maneira radial, mantendo homogênea e constante a temperatura em todos os recipientes.

Para a tomada de temperatura, luz e oxigênio, foram utilizados, respectivamente, um termômetro de vidro com bulbo de mercúrio líquido, um luxímetro marca Lux Meter, mod. LX-101, e um oxímetro, marca Schott, mod. Handylab OX1/SET. O experimento teve início às 18:30h do dia 12 de dezembro de 2003 e se estendeu até às $21: 30 \mathrm{~h}$ do dia seguinte, correspondendo a um tempo total de 27 horas. A cada hora, temperatura e oxigênio dissolvido eram aferidos em todos os frascos (Tabela 1). A irradiância foi verificada de hora em hora, entre às 6:30 e às 19:30h do dia 13 de dezembro. O fotoperíodo para esta época do ano, na latitude de 27ํㅡ é de 14:10.

\section{Análise estatística e percentual de saturação de $\mathrm{O}_{2}$}

Os tratamentos foram comparados através de uma análise de variância unifatorial ANOVAe as médias através do teste Tukey (Zar, 1999). As análises estatísticas foram feitas através do programa "Statistica", versão 99. O percentual de saturação de oxigênio foi calculado através da fórmula sugerida por Kubitza (2003): \% Saturação $\mathrm{O}_{2}=\left(\mathrm{C}_{\mathrm{a}} \cdot \mathrm{C}_{\mathrm{s}}^{-1}\right)$. 100, onde $\mathrm{C}_{\mathrm{a}}$ é a concentração atual e $\mathrm{C}_{\mathrm{s}}$ é a concentração de saturação do oxigênio.

\section{RESULTADOS}

Durante a realização do experimento, o tempo se apresentou chuvoso no decorrer do período noturno, mantendo a temperatura ambiente estável, em torno de $26 \stackrel{\circ}{ } \mathrm{C}$. Durante o dia, o tempo se apresentou com sol pleno, onde a temperatura ambiente ultrapassou os $33^{\circ} \mathrm{C}$. Às 7:30h a intensidade luminosa aferida foi de 807 lux. O período de maior intensidade luminosa foi registrado entre 10:30 e 15:30h, com uma média de 1.216 lux, sendo o horário das 12:30h o pico de máxima intensidade, com 1.424 lux. Durante a tarde, registrou-se para os horários de 16:30, 17:30, 18:30 e 19:30h, as respectivas intensidades de 1.109, 834, 647 e 401 lux.

Observou-se que a ativação do Fotossistema II da alga ocorreu a partir das 6:30h. Em todos os tratamentos com alga foi observado o maior pico de concentração de oxigênio ao final da tarde (médias superiores a $38 \mathrm{mg} \mathrm{de}_{2} \mathrm{~L}^{-1}$ ), entre 18:30 e 19:30h, correspondendo a um percentual de saturação superior a $500 \%$. Isto ocasionou oscilação das medidas observadas no oxímetro, cujas faixas de supersaturação foram superiores à sensibilidade do aparelho.

Constatou-se que a alga, em densidade de $1 \mathrm{~g} \mathrm{~L}$ ${ }^{1}$, manteve uma concentração média de $2,00 \mathrm{mg}$ de $\mathrm{O}_{2}$ $\mathrm{L}^{-1}$ durante a noite. $\mathrm{E}$ seu respectivo controle apontou uma concentração média de $1,87 \mathrm{mg}$ de $\mathrm{O}_{2} \mathrm{~L}^{-1}$. Com relação à produção de fotossíntese, esta densidade algal mostrou uma relativa estabilização do aparelho fotossintético entre 12:30 e 15:30h, onde o valor médio da supersaturação de oxigênio nesta faixa horária foi de $23,26 \mathrm{mg}$ de $\mathrm{O}_{2} \mathrm{~L}^{-1}$. O pico da supersaturação de oxigênio foi registrado às 19:30h, alcançando uma média de $38,17 \mathrm{mg} \mathrm{de}_{2} \mathrm{~L}^{-1}$, correspondente a 564,64\% (Fig. 2a).

A densidade de $2 \mathrm{~g} \mathrm{~L}^{-1}$ apresentou uma ligeira atenuação da produção fotossintética entre 13:30 e 15:30h, com uma média de supersaturação de $31,21 \mathrm{mg}$ 
อิ

ชิ

웡

岀

$\frac{\pi}{\frac{\pi}{\sigma}}$

$\varepsilon$

ब

잉

응

ํํㄹ

㟧

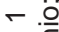

$\frac{\pi}{0} \frac{\sqrt{0}}{0}$ |l 递

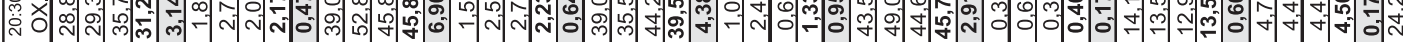

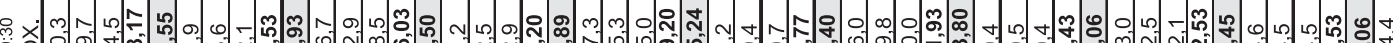

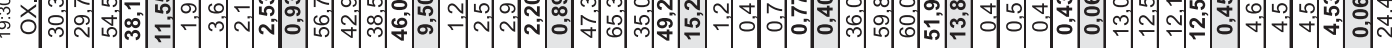
-

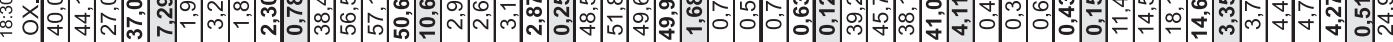
舟 策

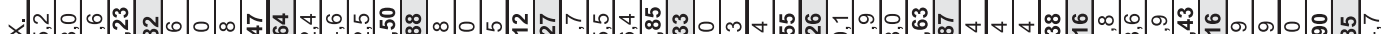
Oֵ

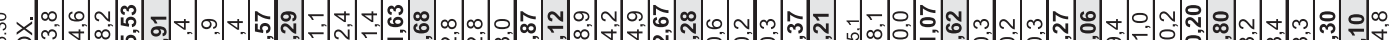

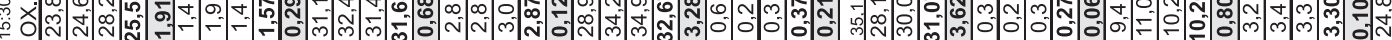
舟

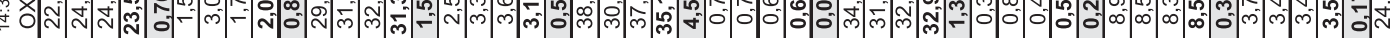

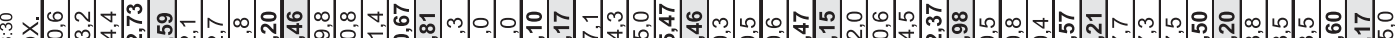

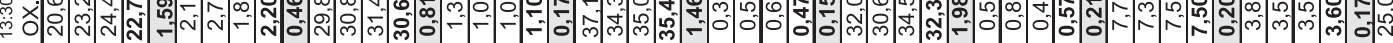

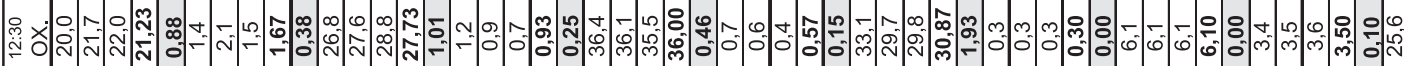

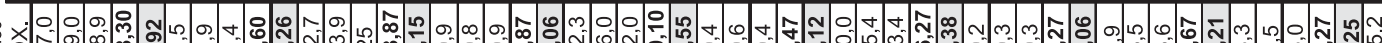

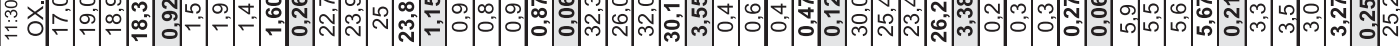
象 OD

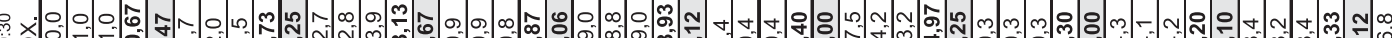
产

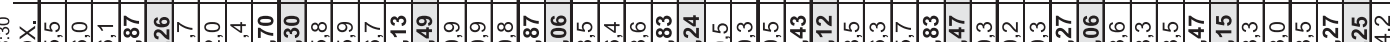

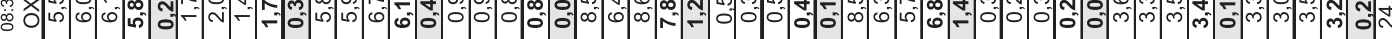

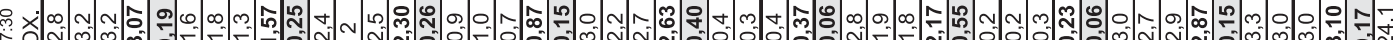

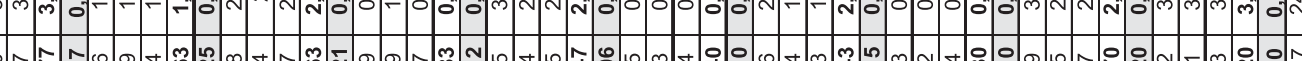

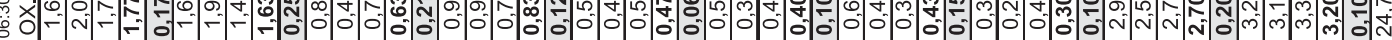

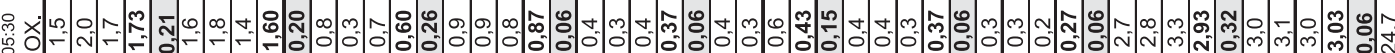
O

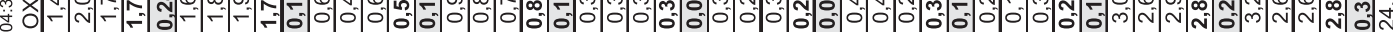
象央

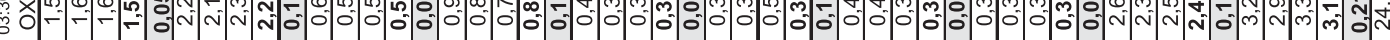
O - 0 -

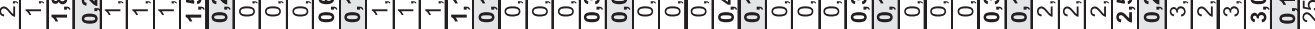

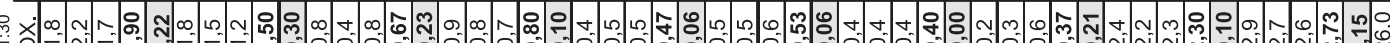

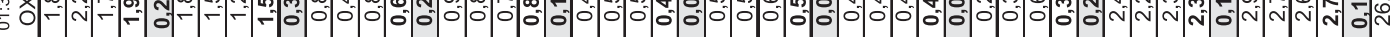
$0 \times 0$ N - $\infty$ N

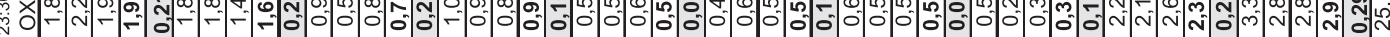

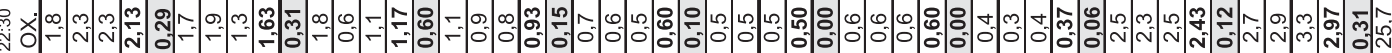
응

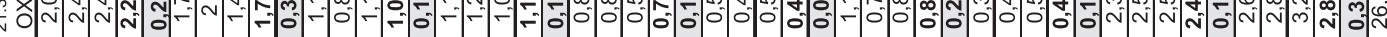
屏

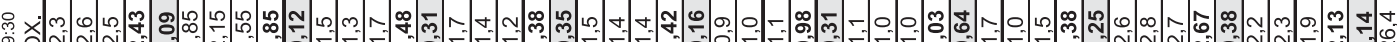

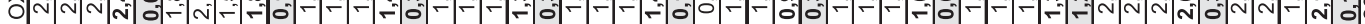

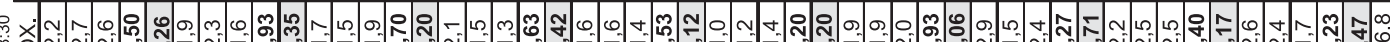

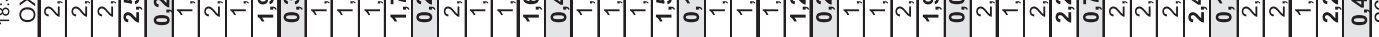

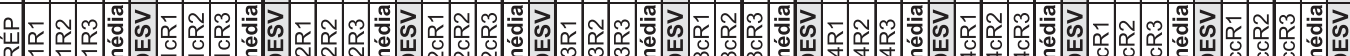
然
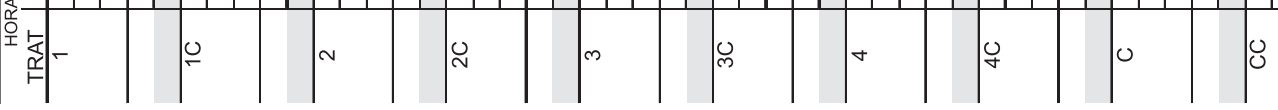

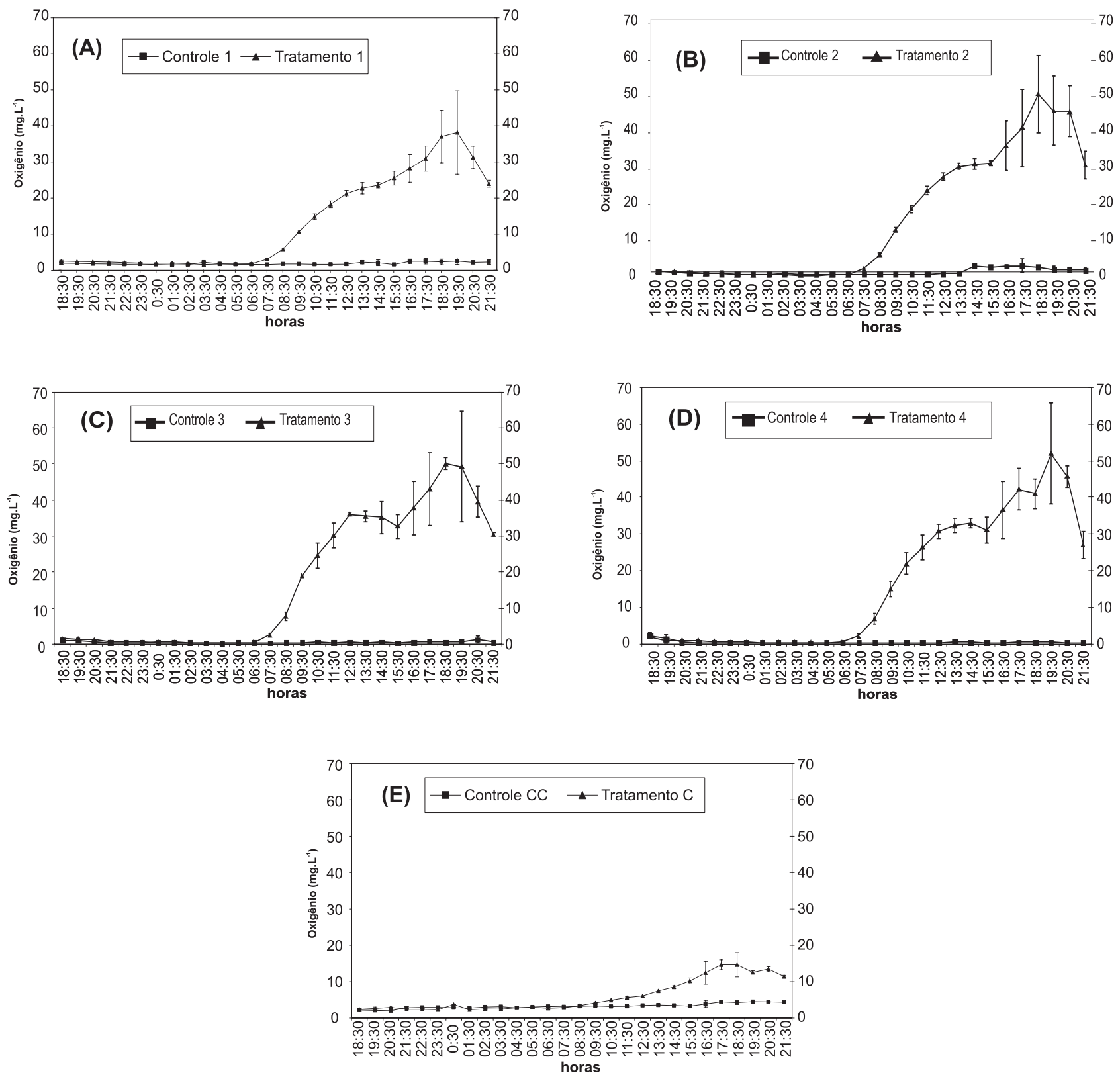

Figura 2 - Relação da demanda respiratória e da produção de oxigênio determinada pela taxa fotossintética da macroalga Ulva lactuca. As barras representam o desvio padrão dos tratamentos e seus respectivos controles. Onde: (a) Densidade algal $=1 \mathrm{~g} \mathrm{~L}^{-1} ;$ (b) Densidade algal $=2 \mathrm{~g} \mathrm{~L}^{-1}$; (c) Densidade algal $=3 \mathrm{~g} \mathrm{~L}^{-1}$; (d) Densidade algal $=4 \mathrm{~g} \mathrm{~L}^{-1}$;(e) Sem alga .

de $\mathrm{O}_{2} \mathrm{~L}^{-1}$. Em seguida, outro crescimento exponencial na concentração de oxigênio foi registrado, atingindo o pico máximo às 18:30h, com supersaturação de $50,67 \mathrm{mg}$ de $\mathrm{O}_{2} \mathrm{~L}^{-1}$, equivalente a $749,55 \%$ (Fig.2b). Durante a noite, a concentração de oxigênio nesta densidade foi mantida em torno de $0,89 \mathrm{mg}$ de $\mathrm{O}_{2} \mathrm{~L}^{-1}$. Seu respectivo controle apontou pequenas oscilações na demanda de oxigênio, registrando-se uma taxa média de $1,47 \mathrm{mg} \mathrm{de}_{2} \mathrm{~L}^{-1}$.

As densidades de 3 e de $4 \mathrm{~g} \mathrm{~L}^{-1}$ tiveram comportamentos muito semelhantes. Em ambos os tratamen- tos se registrou uma concentração média de $0,67 \mathrm{mg}$ de $\mathrm{O}_{2} \mathrm{~L}^{-1}$, no período entre as 18:30 e 6:30h. A produção fotossintética máxima ocorreu, respectivamente, às 12:30h, com concentração de $36,00 \mathrm{mg} \mathrm{de}_{2} \mathrm{~L}^{-1}$ para o tratamento 3 , e às $14: 30 \mathrm{~h}$, com concentração de $32,93 \mathrm{mg}$ de $\mathrm{O}_{2} \mathrm{~L}^{-1}$ para o tratamento 4 . Constatou-se redução destas concentrações, em 9,25\% para o tratamento 3, entre $12: 30$ e $15: 30 \mathrm{~h}$, e em $5,68 \%$ para o tratamento 4, entre 14:30 e 15:30h (Fig. 2c e 2d). Especificamente no tratamento 4 , observou-se outra ligeira queda de $2,84 \%$ na concentração de oxigênio, entre 
17:30 e 18:30h (Fig. 2e). O pico de produção de oxigênio, registrado no tratamento 3 , foi de $49,97 \mathrm{mg}$ de $\mathrm{O}_{2} \mathrm{~L}$ ${ }^{1}(739,20 \%)$, às 18:30h (Fig.2c). Já no tratamento 4 , foi de 51,93mg de $\mathrm{O}_{2} \mathrm{~L}^{-1}(768,20 \%)$, às 19:30h (Fig.2d). Em ambos os controles referentes a estes tratamentos, as taxas de oxigênio se mantiveram constantes ao redor de 0,58 e $0,47 \mathrm{mg}$ de $\mathrm{O}_{2} \mathrm{~L}^{-1}$ nos tratamentos 3 e 4, respectivamente.

O tratamento sem alga obteve incremento de oxigênio, onde se observou uma concentração máxima de 14,68mg de $\mathrm{O}_{2} \mathrm{~L}^{-1}$ às 18:30 (Fig.2e). O controle se manteve constante, apresentando uma concentração média de oxigênio de $3,29 \mathrm{mg}$ de $\mathrm{O}_{2} \mathrm{~L}^{-1}$.

\section{DISCUSSÃO}

Constatou-se com este trabalho que a radiação solar, nos horários próximos ao meio-dia, foi muito intensa, onde os efeitos da radiação incidente sobre $U$. lactuca puderam ser observados através da fotoinibição do aparelho fotossintético da alga. Esta inibição ou inativação ocorrida no Fotossistema II foi constatada em todos os tratamentos com alga, entre 12:30 e 15:30h, embora com menor efeito sobre o tratamento com baixa densidade $\left(1 \mathrm{~g} \mathrm{~L}^{-1}\right)$. Este processo de fotoinibição tem sido reportado por vários autores, através de estudos com outras espécies de algas deste gênero, sugerindo uma redução reversível do transporte de elétrons no Fotossistema II, como forma de proteção do aparelho fotossintético sob irradiação excessiva, após a ocorrência de uma fotossíntese máxima (Coutinho \& Zingmark, 1993; Hernández et al., 1997; Vergara et al., 1997; Grobe \& Murphy, 1998; Altamirano et al., 2000; Altamirano et al., 2000; Cabello-Pasini et al., 2000; Gordillo et al., 2001 e Han et al., 2003). Estudos realizados com $U$. rigida mostraram que a radiação excessiva de UV-B não só interferiu no processo fotossintético da alga como também reduziu em $50 \%$ a taxa de crescimento desta espécie em apenas sete dias (Altamirano et al., 2000). Entretanto, para que ocorra a fotossíntese máxima, é necessária a presença ou absorbância do raio UV-B (Grobe \& Murphy, 1998 e Altamirano et al., 2000).

Nos tratamentos com densidades de 3 e de $4 \mathrm{~g}$ $\mathrm{L}^{-1}$, a redução do oxigênio detectada imediatamente após a fotossíntese máxima, entre 14:30 e 15:30h, sugere uma possível inibição do Fotossistema II, resultado da irradiação excessiva. Este comportamento em relação a radiação incidente tem sido observado nos estudos com U. rigida por Altamirano et al. (2000), onde ocorreu a redução dos valores de $\mathrm{O}_{2}$ em conseqüência da saturação da luz no processo fotossintético. PérezRodríguez et al. (1998) também constataram respostas significativas da macroalga Dasycladus vermicularis
(Chlorophyta) quando exposta a níveis elevados de irradiação ultra-violeta. Segundo estes autores, estas macroalgas produzem substâncias, como os florotaninos, que absorvem na faixa dos raios ultravioleta protegendo o interior da célula contra os efeitos nocivos dos respectivos comprimentos de onda. Estas substâncias são excretadas ou exudadas para o meio, um fenômeno de proteção do mecanismo fotossintético em resposta ao estresse da irradiação solar. Esse fenômeno de excreção ou exudação de florotaninos, induzido pela irradiação UV-B foi observado na macroalga parda Macrocystis integrifolia por Swanson \& Druehl (2002).

As elevadas taxas de supersaturação de oxigênio observadas neste experimento foram decorrentes do impedimento da difusão do $\mathrm{O}_{2}$ da água dos frascos de Erlenmeyer para o ar, devido às dimensões da abertura dos frascos e à cobertura com película de PVC. Os pontos de supersaturação máxima de oxigênio ocorridos em todos os tratamentos com alga, entre 18:30 e 20:30h, podem ter sido favorecidos pela predominância da faixa espectral da luz vermelha, contribuindo para uma melhor absorbância da faixa de $680 \mathrm{~nm}$ pela clorofila a da alga, conferindo melhor atividade do Fotossistema II. Em estudos realizados com U. curvata e U. rotundata, Vergara et al. (1997) constataram que, ao entardecer a reflectância da superfície da água aumenta o ângulo zenital das mudanças de irradiação incidente, favorecendo a predominância de uma faixa espectral vermelha, entre 600 e $700 \mathrm{~nm}$, justamente onde ocorre a absorbância pela clorofila a destas espécies algais. Provavelmente, este fator tenha contribuído no melhor desempenho fotossintético, proporcionando elevadas concentrações de $\mathrm{O}_{2}$ ao final do dia.

Uma outra hipótese que pode justificar a supersaturação de oxigênio ao final da tarde é a ocorrência de um processo de histerese no mecanismo do Fotossistema Il da alga, ou seja, um atraso transitório do efeito fotossintético gerando uma inversão sobre a produção fotossintética. Estudos realizados com Ulva $\mathrm{sp}$. revelaram um aumento significativo das taxas de transporte de elétrons durante a manhã e uma redução das mesmas durante a tarde (Levy et al., 2004). Por outro lado, estes autores constataram que $\circ \mathrm{O}_{2}$ produzido pelas algas foi significativamente maior de tarde que de manhã, sugerindo um processo de histerese (Miza et al. 2006).

A super produção de $\mathrm{O}_{2}$ pelas algas do gênero Ulva ao final do dia ainda é um fato bastante complexo. Lobban \& Harrison (1994) atribuem a supersaturação de oxigênio por essas algas ao final da tarde a uma possível interação com os fluxos de $\mathrm{Mg}^{2+}$. De qualquer forma, $U$. lactuca apresentou, em todas as densidades, uma supersaturação de oxigênio superior em 150\% a supersaturação do fitoplâncton. 
Com relação à respiração, as depleções de oxigênio observadas principalmente nos tratamentos com densidades de 3 e de $4 \mathrm{~g} \mathrm{~L}^{-1}$ são devidas à maior da biomassa algal empregada, bem como das reservas de carbono utilizadas pela alga para reduzir, através do processo fotorrespiratório, o excesso de energia produzida sob altos níveis de irradiação solar. Gordillo et al. (2001) constataram que, em U. rigida, o aumento de $\mathrm{CO}_{2}$ resulta em maior assimilação de $\mathrm{N}$ para produção de sua biomassa através do suprimento de carbono livre, afetando o balanço da relação $\mathrm{C}: \mathrm{N}$ da alga, quando a mesma se encontra sob elevados níveis de irradiação. Segundo Vergara et al. (1997) os valores acumulativos da taxa respiratória em U. curvata e em $U$. rotundata tendem a aumentar no verão e a diminuir no inverno.

As densidades de 1 e de $2 \mathrm{~g} \mathrm{~L}^{-1}$ apontaram meIhores resultados de performance fotossintética, pois a resposta da fotoinibição ao estresse causado pela saturação de raios incidentes foram menos marcantes que nos tratamentos com densidades maiores. $\mathrm{Da}$ mesma forma, os tratamentos com menor densidade algal apresentaram menores taxas respiratórias, resultantes da menor demanda metabólica, proporcionada pela menor biomassa. As densidades 1 e $2 \mathrm{gL}^{-1}$, comparadas ao controle sem alga, incrementaram a demanda de oxigênio em 42 e 56\%, respectivamente, enquanto que as densidades $3 \mathrm{e} 4 \mathrm{gL}^{-1}$, consumiram $81 \mathrm{e}$ $84 \%$ a mais do referido gás, respectivamente (Fig. 2). Nas densidades de 3 e de $4 \mathrm{~g} \mathrm{~L}^{-1}$ ocorreu sobreposição dos talos algais, limitando o acesso à luz. Duke et al. (1986) constataram que a estocagem de Ulva, onde ocorre a sobreposição do material, não aumenta o rendimento da alga devido à limitação da luz.

\section{CONSIDERAÇÕES FINAIS}

Ficou constatado com este estudo que a macroalga Ulva lactuca pode representar um grande potencial de cultivo integrado às fazendas de camarões marinhos em sistema de recirculação, desde que as algas, evidentemente, estejam situadas em uma bacia de estabilização. A supersaturação de oxigênio gerada pela atividade fotossintética desta macroalga superou em muito a sua taxa de respiração. Este fato pode ser perfeitamente explorado para otimizar a disponibilidade de oxigênio dissolvido nos viveiros de camarões marinhos, procedendo-se uma circulação da água ao final do dia. Certamente, este procedimento contribuirá na manutenção, por tempo indeterminado, das faixas ótimas de oxigênio para o camarão, reduzindo o tempo de funcionamento dos aeradores e, conseqüentemente, minimizando os custos de produção através da redução dos gastos de energia elétrica. Com relação à densidade a ser utilizada, apesar dos valores de produção de oxigênio serem mais elevados na densidade de $3 \mathrm{~g} \cdot \mathrm{L}^{-1}$, a elevada demanda respiratória pode proporcionar ambiente anóxico durante a noite, promovendo o estresse da própria população algal empregada. Desta forma densidades menores entre 1 e $2 \mathrm{~g} \cdot \mathrm{L}^{-1}$, além de proporcionarem elevados teores de oxigênio durante o dia, consomem proporcionalmente pouco o referido gás durante a noite, favorecendo a manutenção dos talos.

\section{REFERÊNCIAS BIBLIOGRÁFICAS}

ALTAMIRANO, M.; FLORES-MOYA, A.; CONDE, F. \& FIGUEROA, F. L. 2000. Growth seasonality, photosynthetic pigments, and carbon and nitrogen content in relation to environmental factors: a field study of Ulva olivascens (Ulvales, Chlorophyta). Phycologia, v.39, n.1, p.50-58.

ALTAMIRANO, M.; FLORES-MOYA, A. \& FIGUEROA, F. L. 2000. Long-term effects of natural sunlight under various ultraviolet radiation conditions on growth and photosynthesis of intertidal Ulva rigida (Chlorophyta) cultivated in situ. Botanica Marina, v.43, p.119-126.

CABELLO-PASINI, A.; AGUIRRE-von-WOBESER, E. \& FIGUEROA, F. L. 2000. Photoinhibition of photosynthesis in Macrocystis pyrifera (Phaeophyceae), Condrus crispus (Rhodophyceae) and Ulva lactuca (Chlorophyceae) in outdoor culture systems. Journal of photochemistry and photobiology B: Biology, n.57, p.169-178.

COUTINHO, R. \& ZINGMARK, R. 1993. Interactions of light and nitrogen on photosynthesis and growth of the marine macroalga Ulva curvata (Kützing) De Toni. J. Exp. Mar. Biol. Ecol., n.167, p.11-19.

DUKE, C. S.; LAPOINTE, B. E.; \& RAMUS, J. 1986. Effects of light on growth, RuBPCase activty and chemical composition of Ulva species (Chlorophyta). Journal of Phycology, v.22, p.362-370.

FLORETO, E. A. T.; HIRATA, H.; ANDO, S. \& YAMASAKI, S. 1993. Effects of temperature, light intensity, salinity and source of nitrogen on the growth, total lipid and fatty acid composition of Ulva pertusa Kjellman (Chlorophyta). Botanica Marina, n.36, p.149-158.

GORDILLO, F. J. L.; NIELL, F. X. \& FIGUEROA, F. L. 2001. Non-photosynthetic enhancement of growth by high $\mathrm{CO}_{2}$ level in the nitrophilic seaweed Ulva rigida C. Agardh (Chlorophyta). Planta, n.213, p.6470.

GROBE, C. W. \& MURPHY, T. M. 1998. Solar ultraviolet$B$ radiation effects on growth and pigment composition of the intertidal alga Ulva expansa 
(Setch.) S. \& G. (Chlorophyta). J. Exp. Mar. Biol. Ecol., n.225, p.39-51.

HÄDER, D. P.; PORST, M. \& LEBERT, M. 2000. On site photosynthetic performance of Atlantic green algae. Journal of Photochemistry and Photobiology B: Biology, n.57, p.159-168.

HAN, T.; HAN, Y. S.; KIM, K. Y.; KIM, J. H.; SHIN, H. W.; KAIN, J. M.; CALLOW, J. A. \& CALLOW, M. E. 2003. Influences of light and UV-B on growth and sporulation of the green alga Ulva pertusa Kjellman. Journal of Experimental Marine Biology and Ecology, n.290, p.115-131.

HENLEY, W. J. 1992. Growth and photosynthesis of Ulva rotundata (Chlorophyta) as a function of temperature and square wave irradiance in indoor culture. Journal of Phycology, v.28, p.625-634.

HERNÁNDEZ, I.; PERALTA, G.; PÉREZ-LLORÉNS, J. L.; VERGARA, J. J. \& NIEL, F. X. 1997. Biomass and growth dynamics of Ulva species in Palmones river estuary. Journal of Phycology, n.33, p.764772.

KING, R. J. \& SCHRAMM, W. 1976. Photosynthetic rates of benthic marine algae in relation to light intensity and seasonal variations. Mar. Biol., n.37, p.215-222.

KIRK, J. T. O. 1994. Light and photosynthesis in aquatic ecosystems. 2.ed., Cambridge: Cambridge University Press, 509p.:il.

KUBITZA, F. 2003. Qualidade da água no cultivo de peixes e camarões. Jundiaí: F. Kubitza, 229p.:il.

LAPOINTE, B. E. \& TENORE, K. R. 1981. Experimental outdoor studies with Ulva fasciata Dalile. I. Interaction of light and nitrogen on nutrient uptake, growth, and biochemical composition. J. Exp. Mar. Biol. Ecol., v.53, p.135-152.

LAPOINTE, B. E. \& DUKE, C. S. 1984. Biochemical strategies for growth of Gracilaria tikvahiae (Rhodophyta) in relation to light intensity and nitrogen availability. Journal of Phycology, n.20, p.488-495.

LARTIGUE, J.; NEILL, A.; HAYDEN, B. L.; PULFER, J. \& CEBRIAN, J. 2003. The impact of salinity fluctuations on net oxygen production and inorganic nitrogen uptake by Ulva lactuca (Chlorophyceae). Aquatic Botany, n.75, p. 339-350.
LEVY, O.; DUBINSKY, Z.; SCHNEIDER, K.; ACHITUV, Y.; ZAKAI, D. \& GORBUNOV, M. Y. 2004. Diurnal hysteresis in coral photosynthesis. Marine Ecology Progress Series, v.268, p.105-117.

LOBBAN, C. S. \& HARRISON, J. P. 1994. Seaweed ecology and physiology. Cambridge: Cambridge University Press, 366p.:il.

MEZA, M. E. M., BHAYA, A., KASZKUREWICZ, E. \& COSTA, M. I. S. 2006. "On-off policy and hysteresis on-off policy control of the herbivore-vegetation dynamics in a semi-arid grazing system', Ecological Engineering 28(2), 114-123.

PÉREZ-RODRÍGUEZ, E.; GÓMEZ, I.; KARSTEN, U. \& FIGUEROA, F. L. 1998. Effects of UV radiation on photosynthesis and excretion of UV-absorbing compounds of Dasycladus vermicularis (Dasycladales, Chlorophyta) from southern Spain. Phycologia, v.37, n.5, p.379-387.

SALISBURY, F. B. \& ROSS, C. W. 1992. Plant physiology. 4.ed., Belmont, California (USA): Wadsworth Publishing Company, 682p. :il.

SWANSON, A. K. \& DRUEHL, L. D. 2002. Induction, exudation and the UV protective role of kelp phlorotannins. Aquatic Botany, n.73, p.241-253.

VERGARA, J. J.; PÉREZ-LLORÉNS, J. L.; PERALTA, G.; HERNÁNDEZ, I. \& NIELL, F. X. 1997. Seasonal variation of photosynthetic performance and light attenuation in Ulva canopies from Palmones river estuary. Journal of Phycology, n.33, p.773-779.

VILLARREAL, H.; HINOJOSA, P. \& NARANJO, J. 1994. Effect of temperature and salinity on the oxygen consumption of laboratory produced Penaeus vannameipostlarvae. Comp. Biochem. Physiol., v.108A, n.2/3, p.331-336.

WERLINGER, C. \& ALVEAL, K. 1996. Macroalgas de interés económico: cultivo, manejo, industrialización. In: FERRARIO, M. \& SAR, E. (Eds.) Influencias de factores abióticos en el cultivo de algas. La Plata (Argentina): Universidad Nacional de la Plata, p.199-228.

ZAR, J. H. 1999. Biostatistical analysis. 4.ed. New Jersey: Upper Saddle River.,

Submetido: Abril/2009 Revisado: Dezembro/2009 Aceito: Abril/2010 